\title{
EFFECT OF SEED SIZE ON GERMINATION AND GROWTH OF SEEDLINGS IN WHEAT (TRITICUM AESTIVUM L.)
}

\author{
Fatma Farag Mohamed, Ahmed Salem Issa Buhedma and \\ Saleh Hamuda Saleh \\ Agronomy Dept., Faculty of Agriculture, Omar Al-Mukhtar Univ., El- \\ Baeda-Libya
}

\begin{abstract}
The investigation was conducted at laboratory of cereal technology, Agronomy Department, Faculty of Agricultural, Omer AL-Mukhtar University, EL-Badia, Libya during 2015 /2016 autumn season to study the effect of three wheat cultivars (Kufra, Masry1 and Kazeino) and three size of grains i.e. 1000 -grain weight ranged from 40.3 to $43.6 \mathrm{~g}$. for large size, $32.14-37.22$ medium and 22.14 to $25.33 \mathrm{~g}$. for small size and their interactions on seedling growth characters of wheat. A factorial experimental design in three replications was applied and LSD test were used to mean comperes. The obtained results can be summarized as follows:

Data showed that there were no significant differences among the wheat cultivars and their effect on germination percentage (\%). But there were significant differences among the wheat cultivars on properties of seedlings. Masry1 wheat cultivar surpassed Kufra and Kazeino wheat cultivars in most of seedling growth characters (which gave the lowest number of days from sowing to completely rise of the first, second and third leaves, root and shoot length, seedling fresh and dry weight and leaf area per seedling. The lowest values were recorded by Kufra wheat cultivar.

Results obtained indicated that there was significant effect for seed size on all the studied traits except of germination percent (\%), where large seeds size reported the shortest significant values for day to emergence (first leaf through scabbard, one leaf, two leaves and three leaves emergence), tallest stem and rootlet, heavy fresh and dry weight seedlings, highest leaf area compared with small seed size.

There was no significant effect for the interactions between seed size and the genotypes on germination percent (\%)and germination strong, fresh and dry weight of seedlings stem and rootlet length, while There was significant effect for the interactions on the first and second leaf emergence and leaf area index. Masry1 wheat cultivar with large seeds size surpassed Kufra and Kazeino in the time of leaves emergence and leaf area index.
\end{abstract}




\title{
ت تأثير حجم الحبة على الإنبات ونمو البادرات في القمح Triticum aestivum L. )
}

\author{
فاطمة فرج محمد ، أحمد سالم عيسى بوهدمه و صالح حموده صالح \\ قسم المحاصيل - كلية الزراعة ـ جامعة عمر المختار - البيضاء - ليبيا

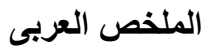

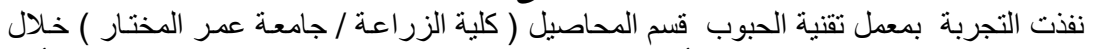

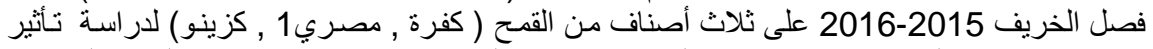

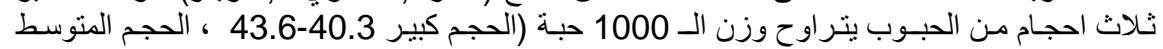

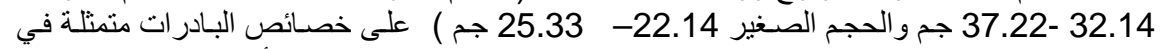

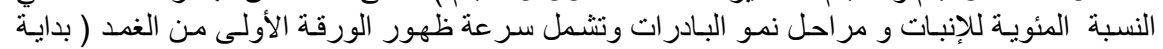

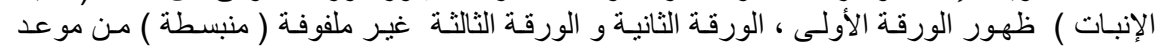

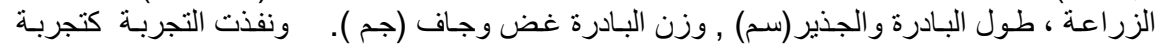

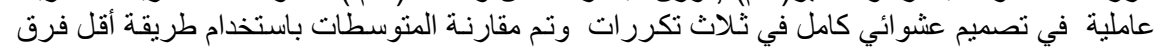

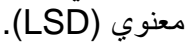

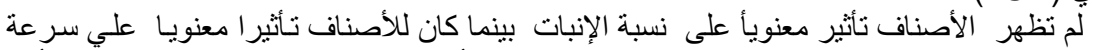

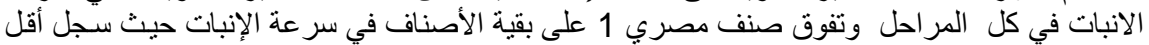

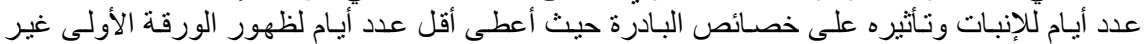

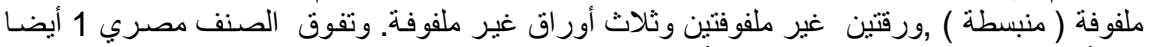

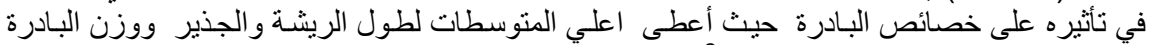

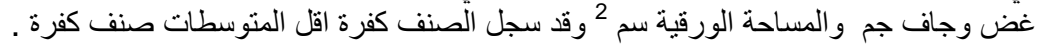

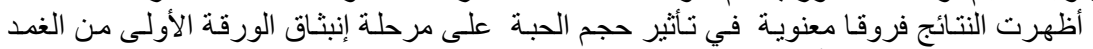

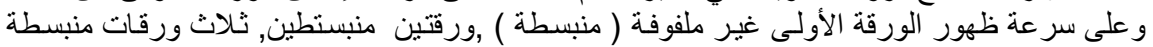

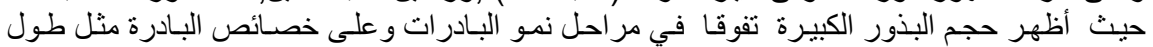

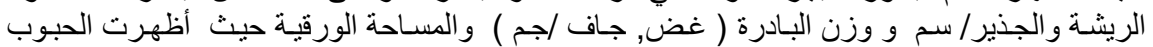

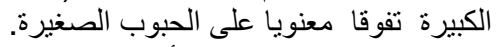

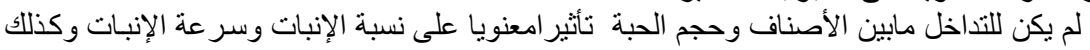

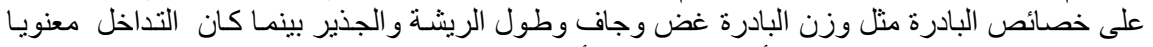

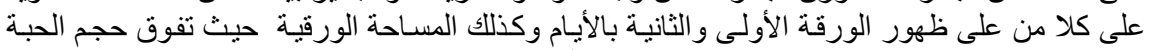

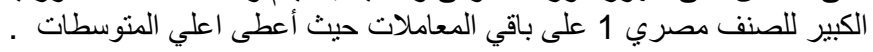

\section{المقدمة}

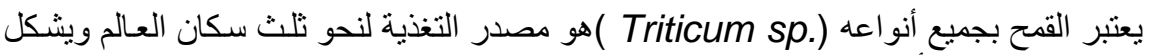

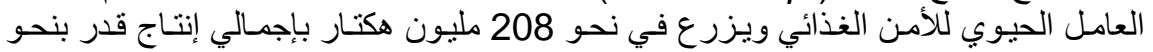

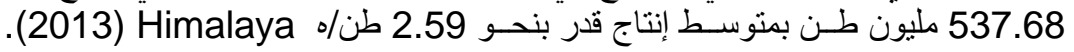

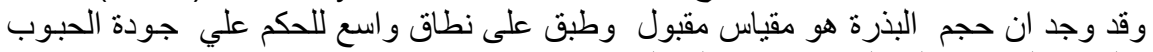
و البذور الكبيرة عالية النمو وقادرة علي البقاء (2004Jerlin and Vadivelu,)

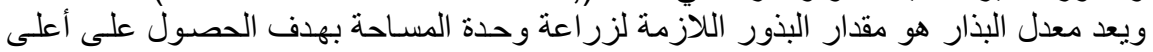

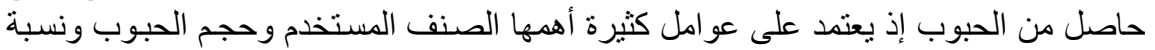

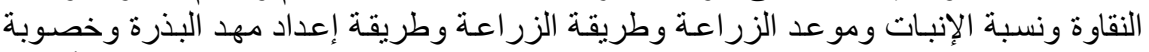

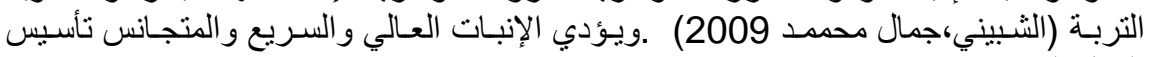

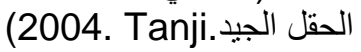




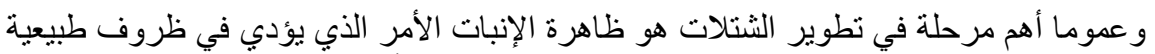

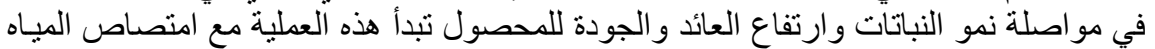

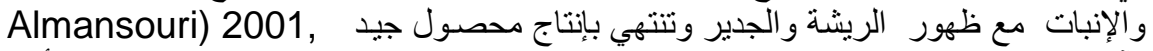

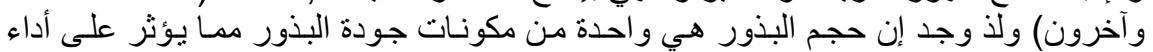

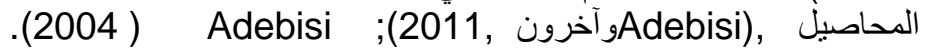

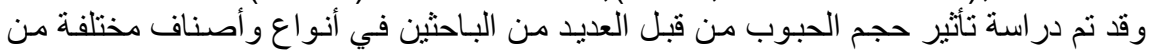

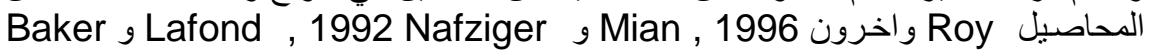

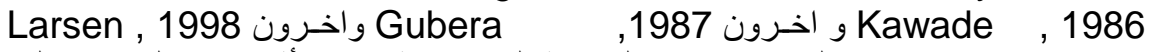

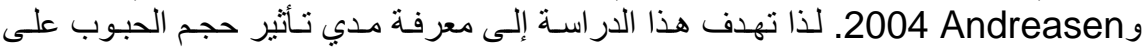

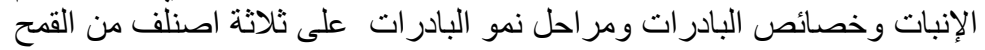

\section{المواد وطرق البحث}

نفذت التجربة بمعمل تقنية الحبوب بقسم المحاصيل ( كلية الزر اعة البح / جامعة عمر المختار

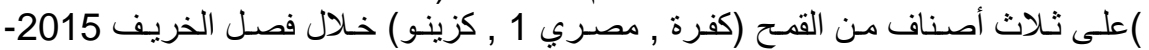

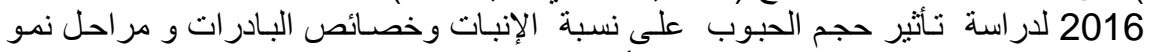

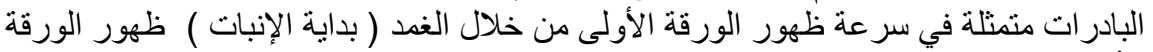

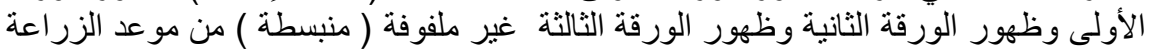

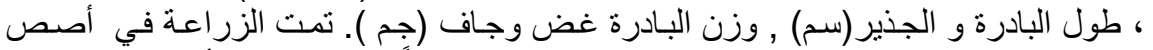

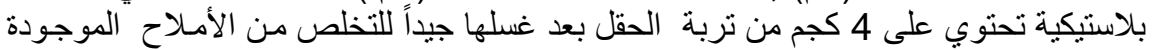

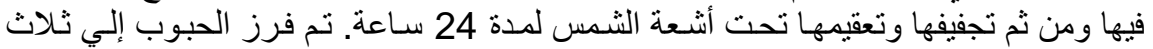

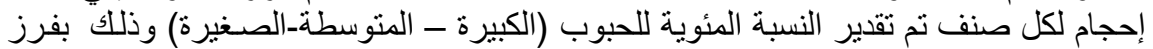

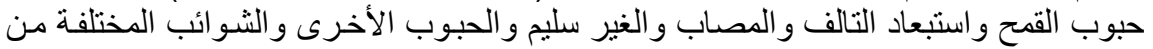

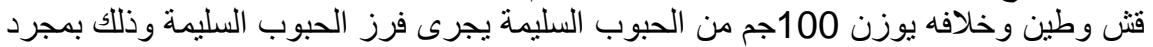

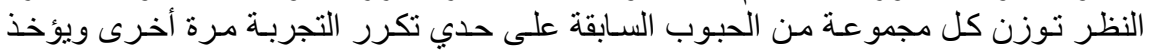

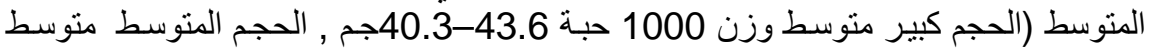

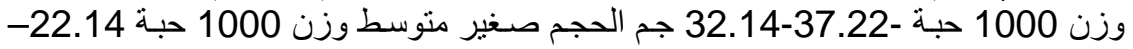

1- اليوم الأول للإنبات ( يوم) هو اليوم الذي حدثت فيه أول حالة إنبات،وان اقل القب القيم تشبر

إلى أسر ع شروع بالإنبات (Shonjani. 2002)

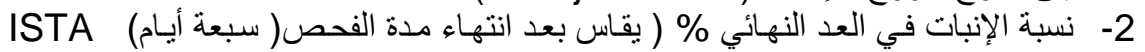

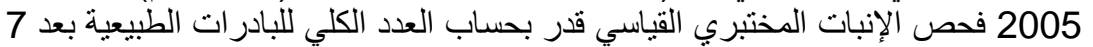

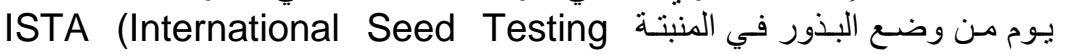
ئ (2005) Association)

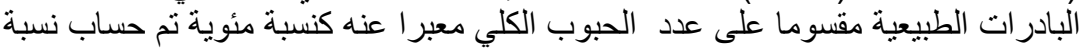

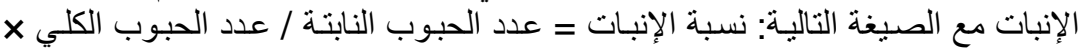

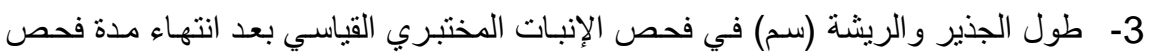

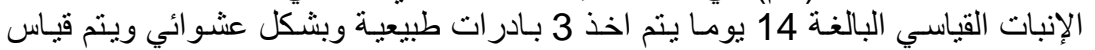

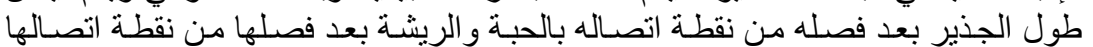

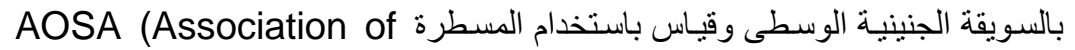

Official Seed Analysts). 1983 
4- الوزن الغض والجاف للبادرة (جم ) تم حسابهما في نهاية فحص الإنبات بعد 14 يوم ،بعد فئس

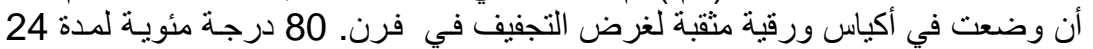

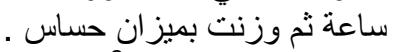

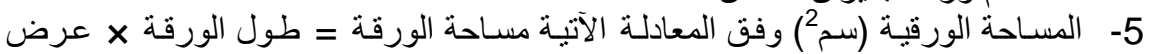
الورقة عند المنتصف ×0.950 (Thomas. 1975)

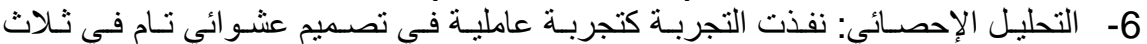

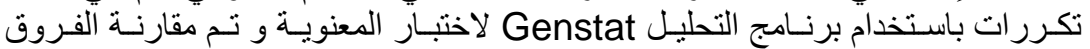

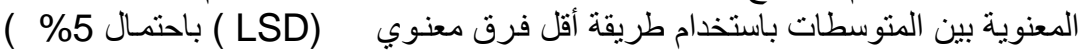
Gomez and Gomez (1984)

\section{النتائج و المناقشة}

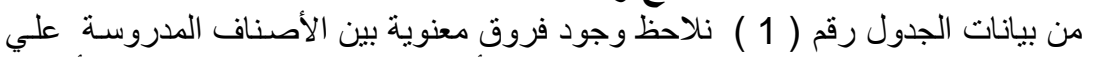

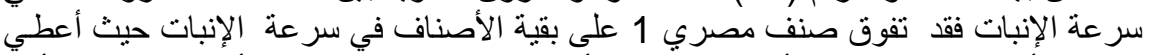

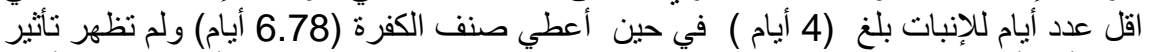

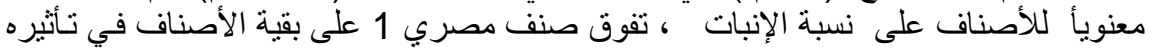

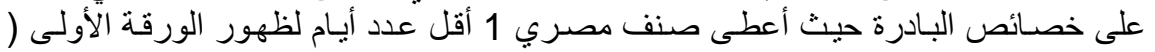
منبسطة ) ,ورقتين , ثناثلث ورقات حيث سجلت القيم (

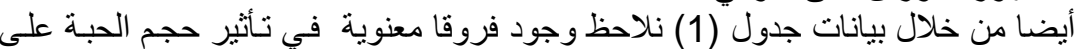

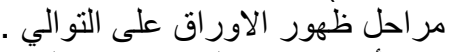

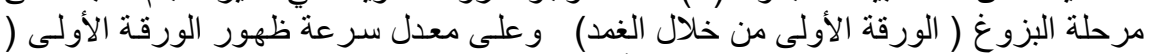

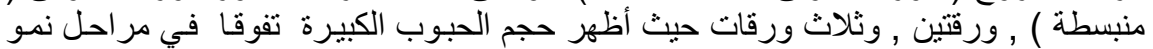

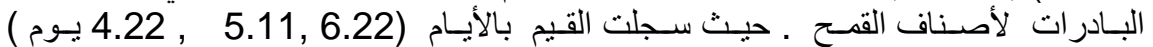

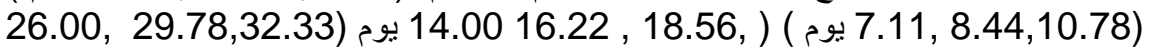

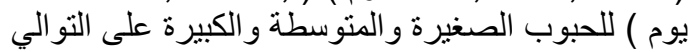

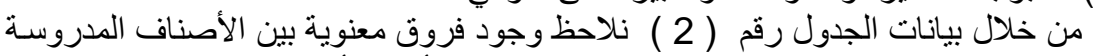

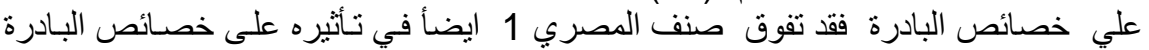

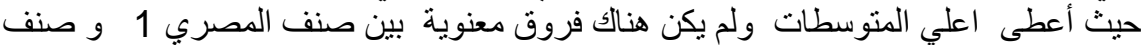

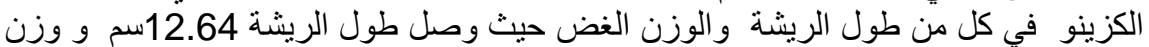

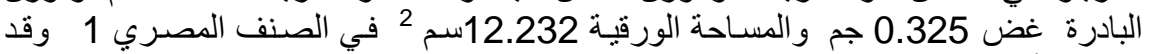

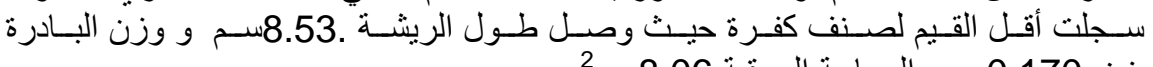

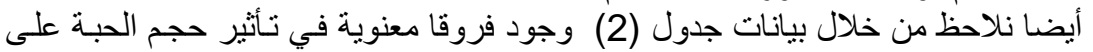

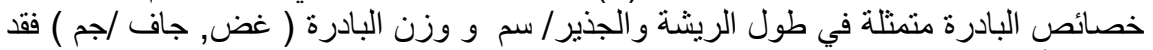

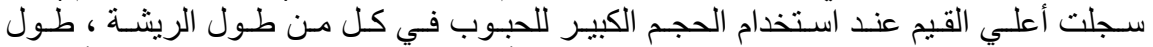

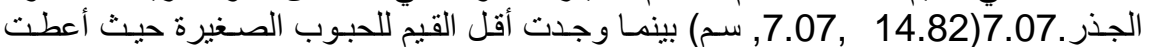

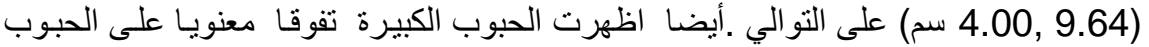

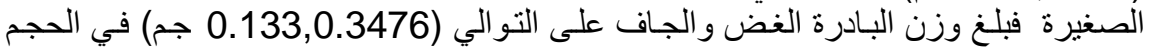

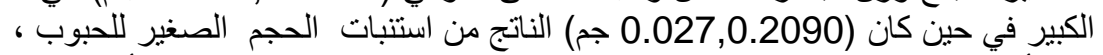

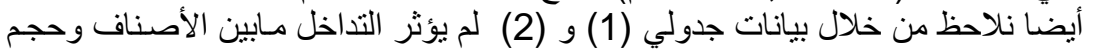

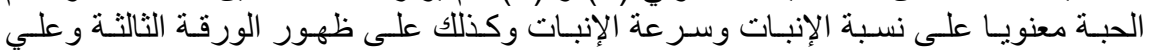

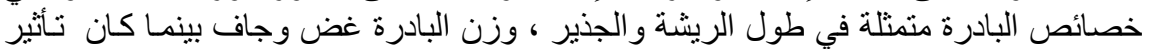

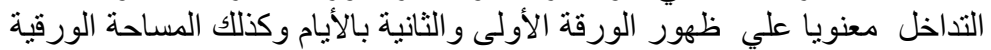

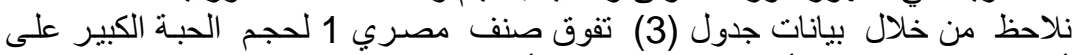

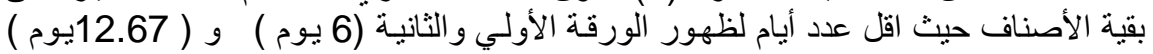




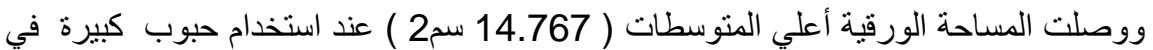

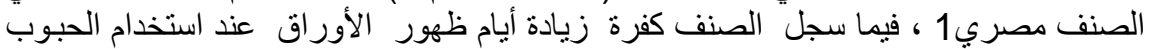

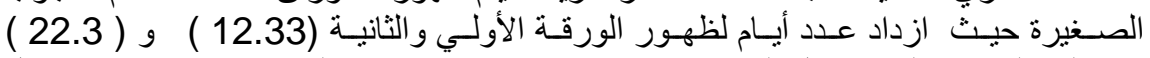

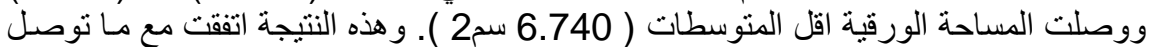

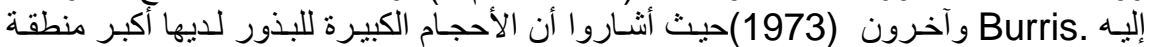
cotyledonary

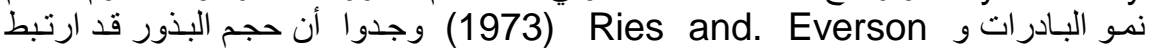

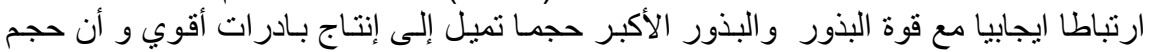

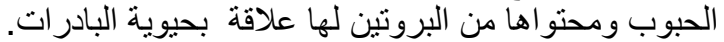

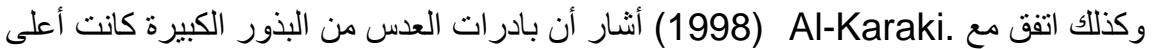

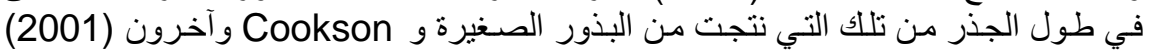

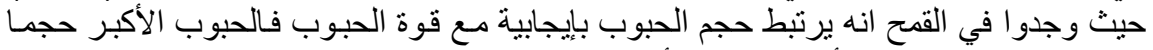

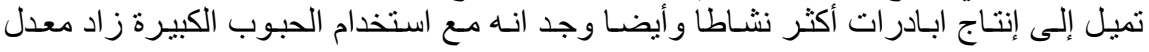

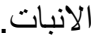
و أيضا Willenborg وآخرون (2005) وجدوا أن حبوب الثوفان الأكبر حجما كانت أعلي

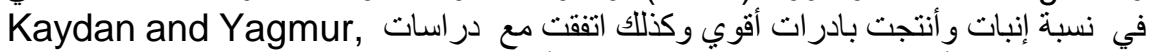
(2008) وجدا أن بذور القرطم الصغيرة كان أقل في امتصـاص المـاء (72\%) من البذور كبيرة (1) (2010). وكذللك (Farhoudi and Motamedi, 2010) ) وجدا أن البذور الكبيرة أنتجت أعلى أنى

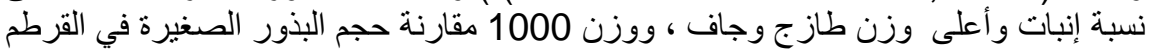

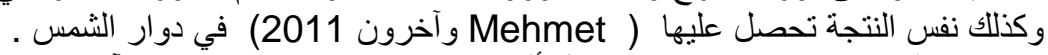

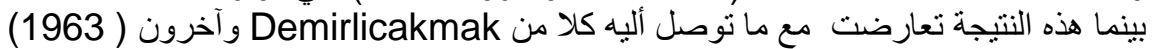

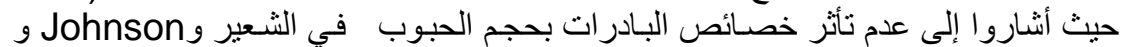
Luedders

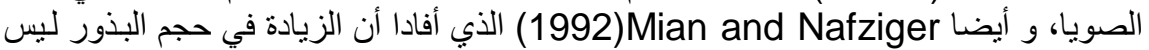

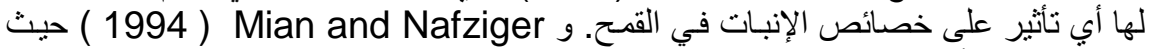
لاحظو ا عدم تأثر نسبة الإنبات بحجم الإنبة في في القئ في القمح الشتوي.

جدول ( 1 ) نأثير الأصناف و حجم الحبة على نسبة الانبات وسرعة ظهور الأوراق في مراحل نمو

\begin{tabular}{|c|c|c|c|c|c|}
\hline & & & & \multicolumn{2}{|c|}{ البادر ات في القمح } \\
\hline ظهور الورقة الثالثة & ظهور الورقة الثانية/ يوم & ظهور الورقةالاولى يوم & |نسبة الإنبات بعد 3 اليام & سرعة الإنبات (يوم ) & المعاملات \\
\hline \multicolumn{6}{|c|}{ الأصناف } \\
\hline 34.44 & 19.22 & 10.56 & 94.5 & 6.78 & كفرة \\
\hline 26.11 & 14.00 & 8.22 & 93.0 & 4.00 & مصري 1 \\
\hline 27.56 & 15.56 & 7.56 & 93.4 & 4.78 & كزينو \\
\hline 1.66 & 0.769 & 0.527 & N.S & 0.72 & $\operatorname{LSD}_{0.05}$ \\
\hline \multicolumn{6}{|c|}{ حجم الحبة } \\
\hline 32.33 & 18.56 & $\begin{array}{ll}0.78 \\
\end{array}$ & 95.4 & 6.22 & حجم صغير \\
\hline 29.78 & 16.22 & 8.44 & 95.6 & 5.11 & حجم متوسط \\
\hline 26.00 & 14.00 & 7.11 & 96.4 & 4.22 & حجم كبير \\
\hline 1.66 & 0.77 & 0.53 & N.S & 0.72 & LSD $_{0.05}$ \\
\hline \multicolumn{6}{|c|}{ التفاعل } \\
\hline N.S & & * & N.S & N.S & LSD $_{0.05}$ \\
\hline
\end{tabular}




\begin{tabular}{|c|c|c|c|c|c|}
\hline الورقية (سم²) & الجاف (جم) & $\begin{array}{c}\text { الوزن (ج) ) } \\
\text { (جض }\end{array}$ & طول الجذير (سم) & |طول الريشة (سم ) & $\begin{array}{l}\text { المعاملات } \\
\text { الصفات }\end{array}$ \\
\hline \multicolumn{6}{|c|}{ الأصناف } \\
\hline 8.059 & 0.040 & 0.171 & 5.39 & 8.53 & كفرة \\
\hline 12.232 & 0.113 & 0.325 & 6.07 & 12.64 & مصري 1 \\
\hline 8.201 & 0.049 & 0.323 & 5.56 & 12.87 & كزينو \\
\hline 0.492 & N.S & 0.023 & N.S & 4.54 & LSD $_{0.05}$ \\
\hline \multicolumn{6}{|c|}{ حجم الحبة } \\
\hline 8.000 & 0.027 & 0.209 & 4.00 & 9.64 & حجم صغير \\
\hline 9.477 & 0.042 & 0.262 & 5.94 & 9.28 & حجم متوسط \\
\hline 11.016 & 0.133 & 0.348 & 7.07 & 14.82 & حجم كبير \\
\hline 0.492 & 0.113 & 0.034 & 0.84 & 4.54 & LSD $_{0.05}$ \\
\hline \multicolumn{6}{|c|}{ التفاعل } \\
\hline * & N.S & N.S & N.S & N.S & LSD $_{0.05}$ \\
\hline
\end{tabular}

جدول (3) تأثير التفاعل بين الأصناف وحجم الحبة على سرعة ظهور الورقة الأولى والثانية والمساحة الورقية

\begin{tabular}{|c|c|c|c|c|}
\hline & & & \multicolumn{2}{|c|}{ لبادرات القمح } \\
\hline المساحة الورقية سم & ظهور الورقة الثانية & ظهورالورقة الاولى & حجم الحبة & الصنف \\
\hline 6.740 & 22.33 & 12.33 & صغير & \\
\hline 8.323 & 19.33 & 10.33 & متوسط & كفرة \\
\hline 9.113 & 16.00 & 9.00 & كبير & \\
\hline 9.487 & 16.00 & 11.33 & صغير & \\
\hline 12.443 & 13.33 & 7.33 & متوسط & مصري 1 \\
\hline 14.767 & 12.67 & 6.00 & كبير & \\
\hline 7.773 & 17.33 & 8.67 & صغير & \\
\hline 7.663 & 16.00 & 7.67 & متوسط & كزينو \\
\hline 9.167 & 13.33 & 6.33 & كبير & \\
\hline 0.853 & 1.33 & 0.91 & & \\
\hline
\end{tabular}

المراجع العربية - ماجية

الشبيني، جمال محمد ـ 2009 ـ تقتيات زراعة و إنتاج القمح ـ المكتبة المصرية للطبة الطباعة والنشر

والتوزيع مركز ـ البحوث الزرعية ع ص500 ـ

المراجع الأجنبية

Adebisi, M.A., 2004. Variation, stability and correlation studies in seed quality and yield components of sesame (Sesamum indicum L.). Ph.D. Thesis, Univ. of Agriculture, Abeokuta, Nigeria.

Adebisi, M.A., T.O. Kehinde, M.O. Ajala, E.F. Olowu and S. Rasaki, 2011. Assessment of seed quality and potential longevity in elite tropical soybean (Glycine max L.) Merrill grown in Southwestern Nigeria. Niger. Agric. J., 42: 94-103.

Al-Karaki, G.N., 1998.Seed size and water potential effects on water uptake, germination and growth of lentil. J. Agron. Crop Sci., 181(4): 237-242.

Almansouri, M., J.M. Kinet and S. Lutts, 2001. Effect of salt and osmotic stresses on germination in durum wheat (Triticum durum Desf.). Plant Soil., 231: 243-254.

AOSA, (Association of Official Seed Analysts).( 1983). Seed Vigour Testing Handbook. Contribution No. 32 to Handbook on Seed Testing Association of Official Seed Analysts, Lincoln, NE, USA. pp. 88 
Burris, J.S., O.T. Edje and A.H. Wahab, 1973. Effects of seed size on seedling performance in soybeans. II. Seedling growth and photosynthesis and field performance. Crop Sci., 13: 207-210.

Cookson, W.R., J.S. Rowarth and J.R. Sedcole, 2001. Seed vigour in perennial ryegrass (Lolium perenne L.): Effect and cause. Seed Sci. Technol., 29: 255-270.

Demirlicakmak, A., M.L. Kaufmann and L.P. Johnson, 1963. The influence of seed size and seeding rate on yield and yield components of barley. Can. J. Plant Sci., 43: 330-337.

Farhoudi, R. and M. Motamedi, 2010.Effect of salt stress and seed size on germination and early seedling growth of safflower (Carthamus tinctorius L.). Seed Sci. Technol., 38: 73-78.

Gomez, K.A. and A.A. Gomez., (1984). Statistical Procedure for Agricultural Research. John Wiley and Sons.

Guberac, V., J. Martincic and S. Maric, 1998. Influence of seed size on germinability, germ length, rootlet length and grain yield in spring oat. Bodenkultur, 49: 13-18.

Himalaya, S. (2013). Wheat weed identification and management under cereal production system. J. Sustainable Soc., 2(3):74-85.

ISTA (International Seed Testing Association).( 2005). International Rules for Seed Testing. Adopted at the Ordinary Meeting.2004, Budapest, Hungary to become effective on 1st January 2005.The International Seed Testing Association. (ISTA).

Jerlin, R. and K.K. Vadivelu, 2004.Effect of fertilizer application in nursery for elite seedling production of Pungam (Pongamiapinnata L. Picrre). J. Trop. Agric. Res. Extension, 7: 69-71.

Johnson, D.R. and V.D. Luedders, 1974. Effect of planted seed size on emergence and yield of soybeans (Glycine $\max$ (L.)Merr.).Agron. J., 66: 117-118.

Kawade, R.M., S.D. Ugale and R.B. Patil, 1987. Effect of seed size on germination, seedling vigour, and test weight of pearl millet. Seed Res., 15: 210-213.

Kaydan, D. and M. Yagmur, 2008.Germination, seedling growth and relative water content of shoot in different seed sizes of triticale under osmotic stress of water and $\mathrm{NaCl}$. Afr. J. Biotechnol., 7: 2862-2868.

Lafond, G.P. and R.J. Baker, 1986. Effects of temperature, moisture stress, and seed size on germination of nine spring wheat cultivars. Crop Sci., 26: 563-567.

Larsen, S.U. and C. Andreasen, 2004. Light and heavy seeds differ in germination percentage and mean germination thermal time. Crop Sci., 44: 1710-1720.

Mehmet, K.D., B. Suay, K. Gamze and U. Oguzhan, 2011. Seed vigor and ion toxicity in safflower (Carthamus tinctorius L.) seedlings produced by various seed sizes under $\mathrm{NaCl}$ stress. Arch. Biol. Sci., 63: 723-729. 
Mian, A.R. and E.D. Nafziger, 1992. Seed size effects on emergence head number, and grain yield of winter wheat. J. Prod. Agric., 5: 265268.

Mian, A.R. and E.D. Nafziger, 1994.Seed size and water potential effects on germination and seedling growth of winter wheat. Crop Sci., 34: 169-171.

Ries, S.K. and E.H. Everson, 1973. Protein content and seed size relationships with seedling vigour of wheat cultivars. Agron. J., 65: 884-886.

Roy, S.K., A.S. Hamid, M. Giashuddin Miah and A. Hashem, 1996. Seed size variation and its effects on germination and seedling vigour in rice. J. Agron. Crop Sci., 176: 79-82.

Sadeghi, H., F. Khazaei, S. Sheidaei and L. Yari, 2011.Effect of seed size on seed germination behavior of safflower (Carthamus tinctorius L.). J. Agric. Biol. Sci., 6: 5-8.

Shonjani, S., (2002). Salt Sensitivity of Rice, Maize, Sugar Beet, and Cotton during Germination and Early Vegetative Growth. Ph.D. Dissoretion, Justus Liebig University Gies- sen.pp.164.

Thomas, H. (1975). The growth response of weather of simulated vegetative swards of single genotype of Lolium perenne. J. Agric. Sci. Camb. 84: 333-343.

Willenborg, C.J., J.C. Wildeman, A.K. Miller, B.G. Rossnaged and S.J. Shirtliffe, 2005. Oat germination characteristics differ among genotypes, seed sizes, and osmotic potentials. Crop Sci., 45: 2023-2029. 